\title{
Effects on the brain of a psychological treatment: cognitive remediation therapy
}

\author{
Functional magnetic resonance imaging in schizophrenia
}

TIL WYKES, MICHAEL BR AMMER, JOHN MELLERS, PETER BRAY, CLARE REEDER, CLARE WILLIAMS and JULIA CORNER

\begin{abstract}
Background The results of one randomised control trial testing a psychological rehabilitation programme aimed at information processing strategies showed improvements in cognition posttreatment.
\end{abstract}

\begin{abstract}
Aims To determine whether there are concomitant brain activation changes as a result of engaging in cognitive remediation therapy (CRT).
\end{abstract}

\begin{abstract}
Method Three groups (patients receiving control therapy or CRTand a healthy control group) were investigated in a repeated measures design using the two-back test. Functional magnetic resonance imaging ( $\mathrm{fMRI}$ ) data and a broad assessment of executive functioning were completed at baseline and posttreatment. Brain activation changes were identified after accounting for possible task-correlated motion artefact.
\end{abstract}

Results $\mathrm{fMRI}$ analyses indicate that the control group showed decreased activation but the two patient groups showed an increase in activation over time. The patient group that received successful CRT had significantly increased brain activation in regions associated with working memory, particularly the frontocortical areas.

Conclusions This is the first time that brain activation changes in a seriously disabled group of patients with schizophrenia can be associated clearly with psychological rather than pharmacological therapy.

Declaration of interest This work was supported by the WellcomeTrust.
Cognitive deficits were described by Kraepelin and Bleuler as 'the core deficit' in schizophrenia but recent evidence suggests that such deficits in processing information may not be refractory but are open to novel interventions collectively called 'cognitive remediation therapy' (CRT) (Wykes et al, 1999; Hogarty \& Flesher, 1999). The mechanism for cognitive improvement following CRT is not well understood but may be related to changes in specific aspects of brain function or to global changes, at a behavioural level, perhaps attributable to non-specific changes in arousal. Alternatively, novel strategies may be adopted to improve overall processing which are reflected in the neural mechanisms involved in completing a task. For example, Wykes (1998) showed that two people who received intensive CRT both improved their performance on a test of cognitive flexibility (verbal fluency) after treatment but by two entirely different information processing strategies that were reflected in their differing brain activation patterns following CRT. The focus of this study is the working memory system, because performance on tasks of working memory has been shown to be related to functioning. Previous studies using positron emission tomography have identified shortterm storage buffers in temporoparietal cortex, whereas attentional and executive components may be subserved by connections between thalamus, cingulate and dorsolateral prefrontal cortex (DLPFC). All studies seem to show activation in the prefrontal cortex (Smith \& Jonides, 1998), suggested to show an inverted ' $U$ ' function of activation in response to increasing memory load (Callicott et al, 1999). In schizophrenia, several functional imaging studies have shown a failure to activate the DLPFC and anterior cingulate during tasks in which healthy volunteers show such activation (Weinberger et al, 1986; Rubin et al, 1994a,b; Kindermann et al, 1997). Studies of working memory have been able to identify a neural network involving anterior cingulate, DLPFC, parietal cortex and precuneus (Mellers et al, 1995), each of which could be assigned a cognitive role in attentional, executive or short-term mnemonic processes for performance of the task. The current study investigated brain activation using functional magnetic resonance imaging (fMRI) at two time points (before and after receiving psychological therapy) on a task involving verbal working memory that is known to be predictive of future functioning. Practice on such tasks has been shown to decrease activation in normal participants. In contrast, our hypothesis was that following psychological therapy patients with schizophrenia should increase their activation in those areas associated with successful task performance because of increased concentration and attention as a result of these forms of rehabilitation. Following Wexler et al (2000) we hypothesised that these areas will specifically include the inferior frontal cortex. We also hypothesised that those people who receive CRT will show greater improvements because the intervention is specifically designed to improve information processing strategies used in working memory tasks. The specificity of the brain activation changes was explored in relation to the behavioural changes noted in the neuropsychological tests.

\section{METHOD}

\section{Design}

The study employed a repeated measures design with three groups (healthy controls and patients with schizophrenia receiving either CRT or control therapy involving intensive occupational therapy activities). The repeated measures were brain images produced by an fMRI activation paradigm and cognitive testing for all groups. For both patient groups, social functioning and symptoms were assessed as part of a more extensive assessment.

\section{Participants}

At the time of the study there were ethical restrictions on the use of fMRI with women, therefore the study concentrated only on men. Twelve men were drawn from consecutive recruits in an ongoing randomised control trial of CRT: six from the CRT group and six from the control therapy group. All patients were recruited from the community psychiatric services covering a 
large geographical area in south London. They fulfilled DSM-IV (American Psychiatric Association, 1994) criteria for schizophrenia and also:

(a) had been in touch with services for at least 2 years;

(b) showed evidence of cognitive difficulties and social functioning problems;

(c) showed no evidence of organic brain disease and no primary diagnosis of substance misuse;

(d) had no plans to change medication during the treatment phase.

In addition, six healthy men with no history of psychiatric disorder were recruited from the local community. All participants gave written consent to take part in the study after the procedures had been fully explained. The groups did not differ in mean age (CRT group: 35 years; patient control: 36 years; healthy control: 36 years) and there were no differences between groups in the mean number of years of formal education $(13,12$ and 12 years, respectively). The patients were a chronic group with more than two-thirds having been in contact with the psychiatric services for more than 10 years.

\section{Therapies}

Both therapies consisted of 40 one-hour individual sessions over a 12 -week period. The control therapy sessions consisted of occupational therapy activities such as role play, making a life diary and relaxation. In the CRT group, the sessions consisted of paper and pencil tasks that were used to practise information processing strategies in three executive functions: cognitive flexibility, working memory and planning. For the memory sessions the therapist included tasks on which information has to be manipulated in memory. The therapist would encourage the use of efficient information processing strategies, including the chunking of information and the use of mnemonic strategies for successful completion of these tasks. Further details are given in Wykes et al (1999).

\section{Medication}

All patient participants were maintained on antipsychotic medication throughout the course of the study and there was little change over the course of treatment in the type or dosage of the medication prescribed. At baseline, nine participants were prescribed typical antipsychotic medication (four on CRT and five on control therapy) and the remainder were prescribed newer antipsychotic medication. At posttreatment one person had changed the type of drug (from typical to atypical in the CRT group) and one person had been transferred from risperidone to clozapine (control therapy). The average dose for the typical antipsychotic medication was $643 \mathrm{mg} /$ day chlorpromazine equivalents (Foster, 1989). The percentage of maximum dose for the atypicals was $64 \%$. At the end of treatment four patients (two in each group) were taking an increased dose of their typical antipsychotic medication. There were no significant differences between groups in the levels of prescribed medication.

\section{Neuropsychological tasks}

Detailed descriptions of these tasks are given in Wykes et al (1999).

\section{Cognitive flexibility}

(a) Hayling Sentence Completion Task (Burgess \& Shallice, 1996).

(b) Controlled Oral Word Fluency Test (Spreen \& Benton, 1977).

(c) Stroop Neuropsychological Screening Test (Trenerry et al, 1989).

\section{Memory}

(a) Visual span: participants are required to reproduce increasingly complex figures presented on a grid from memory. The key measure is the highest level at which two out of four figures are correctly recalled.

(b) Sentence span: this is based on the Daneman \& Carpenter (1980) Sentence Span task. Groups of sentences are read to the participant, who must recall the last word in each sentence after hearing the whole group. The number of sentences within a group increases throughout the test and the span is the number of sentences in the group where the last word was recalled.

(c) Digit span: from the Wechsler Adult Intelligence Scale - Revised (Wechsler, 1981).

(d) Dual Span (Della Sala et al, 1995): measures Baddeley's (1986) conception of working memory as the ability to process two tasks at the same time (in this version, tracking and remembering numbers). The measure of working memory is defined by a formula produced by Baddeley \& Della Sala (1996), which combines performance on both the memory and tracking tasks.

\section{Procedure}

Each patient first consented to be in the CRT trial and, after baseline assessment, each was randomised to either the CRT group or the control therapy group. They were then asked to take part in the scanning project. If they agreed, and were also able to complete the activation task (the twoback task) with at least an $80 \%$ accuracy rate outside the scanner, they were included in the current study. Following the 12-week therapy window the two patient groups completed the second set of scans. The healthy control group also completed the scans with a 3-month interval. All groups completed cognitive performance tasks at the time of the scanning procedure.

\section{Functional magnetic resonance imaging}

\section{Cognitive activation task}

The task adopted in this study was the ubiquitous $n$-back task in which the participant has to identify whether a visually presented letter had appeared before. The load chosen for this study was two-back because it has been shown to be a relatively easy task for our patients to perform with high accuracy. Callicott et al (1999) have suggested that individuals may disengage from the task if it is too difficult, and so reasonable accuracy on the task was thought to be important rather than controlling for capacity constraints. The task involves continuously updating a record of the previous two letters into a short-term store and then comparing the current letter on the screen with the contents of that store. These are the cognitive operations in working memory described by Baddeley (1986). The activation during this task was compared with the activation in a vigilance condition that controlled for the perception of the letters as well as the motor response to targets. The two tasks are matched for target frequency. The activation paradigm used for this study is based on that described by Cohen et al (1994), and further developed by Mellers et al (1995), and consists of three conditions.

(a) Working memory condition (task): here participants view a random series of letters and are asked to indicate, by a 
button press with a finger of the right hand, when the letter on the screen is the same as that occurring two letters previously (e.g. B-G-B, but not B-GL-B or B-B).

(b) Vigilance condition (look for X): participants view a series of letters presented in alphabetical order and are asked to respond when the letter $\mathrm{X}$ appears out of sequence (e.g. A-B-X).

(c) Baseline condition: for this, participants simply view a blank screen.

The beginning of each condition is indicated to the participant by a message on the screen ('Look for X' or 'Task') and participants are asked to say each letter to themselves (subvocally) as it appears on the screen in both the vigilance and working memory conditions. The rate of letter presentation (one every $1.2 \mathrm{~s}$ ) and the frequency of target responses (two or three in each 15-letter epoch) are the same in the vigilance and working memory conditions. Both of these conditions therefore required participants to attend to the screen, to encode the letters and to deliver a specified motor response.

The working memory condition differed from the vigilance condition in that it required participants to keep a continuously updated record of the previous two letters and to compare the current letter on the screen with that occurring two before. The fMRI experiment described here involved a comparison of working memory and the vigilance conditions performed by each participant while they were in the scanner. The two conditions alternated ten times over a 5-min imaging period, giving five contrasting 'blocks'. Each condition ran for 30-s epochs, during which image volumes, each consisting of ten axial slices, were acquired.

\section{Image acquisition}

Magnetic resonance images were acquired using a 1.5-Tesla General Electric Sigma system (General Electric, Milwaukee, USA) fitted with ANMR hardware and software (Advanced Nuclear Magnetic Resonance, Woburn, Massachusetts, USA). A quadrature birdcage head coil was used for radiofrequency transmission and reception. Foam padding within the head coil and a restraining forehead band minimised head movement throughout the scanning procedure. Using a midsagittal scout image, ten axial slice positions ( $5 \mathrm{~mm}$ thick, $0.5 \mathrm{~mm}$ interslice gap) were orientated in the anterior commissureposterior commissure (AC-PC) plane and the fourth slice was positioned on the AC-PC plane. For each participant, 100 $\mathrm{T}_{2}{ }^{*}$-weighted multi-slice data-sets depicting blood-oxygenation-level-dependent (BOLD) contrast were acquired by use of a gradient echo echoplanar technique (echo time $=40 \mathrm{~ms}$; repetition time $=3000 \mathrm{~ms}$ ).

\section{Image analysis}

Image preprocessing. Prior to brain activation mapping, the effects of subject motion were minimised by a threedimensional re-alignment and regression procedure at the individual level and correlated at group level by inclusion as a covariate in the analysis. This procedure has been described previously in detail by Bullmore et al (1999).

Brain activation mapping. Periodic change in $T_{2}{ }^{*}$-weighted signal intensity at the experimentally determined frequency of alternation between OFF and ON conditions were estimated by an iterated least-squares fit of a sinusoidal regression model to the movement-corrected time series observed at each voxel. This model included a pair of sine and cosine terms at the fundamental frequency of the OFF/ON conditions, represented by the coefficients $\gamma$ and $\delta$. The sign of $\gamma$ indicates the timing of the periodic response signal in relation to the OFF/ON conditions. If $\gamma>0$, then the fitted model response indicates that a maximum signal is observed during the $\mathrm{ON}$ condition, whereas if $\gamma<0$ the model indicates the maximum signal during the OFF condition. The power of the ON/OFF response frequency was estimated by $\gamma^{2}+\delta^{2}$ and divided by its standard error, giving a standardised test statistic entitled the fundamental power quotient (FPQ). The FPQ was computed at each voxel. Significantly activated voxels (with a probability of a false-positive activation $<10^{-3}$ ) were identified by spatiotemporal randomisation testing (Brammer et al, 1997). These were coloured and superimposed on the mean grey-scale (echoplanar) image to yield a brain activation map for each participant (Bullmore et al, 1996). Generic brain activation maps were constructed following the method set out in Brammer et al (1997).

\section{Overall statistical analysis}

Because of the small sample size and the possibility of type II errors, the analysis set out to identify only the largest differences. Small but significant differences may have been present but these were not the focus of the study. The statistical analysis of the brain imaging data was carried out as follows. First, cortical areas were identified which were of relevance to the memory component of the task, the perception of the target and the motor response (i.e. the vigilance $v$. working memory condition). These regions of interest were identified using analyses of covariance following the method described in Curtis et al (1999). The coordinates of these brain areas were then checked against the generic brain activation map for normal control data and were chosen for further analysis if they had a maximum FPQ of at least 1.9 (voxel-wise type I error probability $<0.00001)$. The FPQ values for each of these previously identified regions were then used in all subsequent analyses: a doubly multivariate repeated measures analysis with 'group' as the between-subject factor (healthy controls, control therapy patients and CRT patients) and 'time' and 'brain region' as the repeated measures within-subject factors for testing all brain regions simultaneously; and if this analysis indicated differences, then these were investigated by looking at the univariate test measures separately to distinguish the differences.

In addition to these analyses, participants were rated as to whether they had made significant gains on the memory tests other than that used in the fMRI experiments. If significant gains were made (improvements across all tests), then they were labelled as a CRT-successful group and their data were compared with those of the remaining participants in a similar way to the analyses described above.

\section{RESULTS}

\section{Clinical and behavioural data}

\section{Baseline data}

Premorbid IQ as measured by the National Adult Reading Test (NART; Nelson \& Willison, 1991) was not significantly different between the groups and although the patient participants had a lower IQ there was a great deal of overlap (mean IQ: CRT group 108; control therapy 102; healthy controls 114). The patient participants performed significantly worse than the healthy controls on all the neuropsychological tests but there were no differences 
Table I Neuropsychological data for all participants at baseline and post-treatment

\begin{tabular}{|c|c|c|c|c|c|c|c|c|}
\hline & \multicolumn{3}{|c|}{ Cognitive remediation group $(n=6)$} & \multicolumn{3}{|c|}{ Control therapy group $(n=6)$} & \multicolumn{2}{|c|}{ Normal controls $(n=6)$} \\
\hline & Baseline & Post-treatment & Improved' & Baseline & Post-treatment & Improved' & Baseline & Post-treatment \\
\hline \multicolumn{9}{|l|}{ Cognitive flexibility tasks } \\
\hline Hayling & 15.8 & 8.0 & $100 \%$ & 10.3 & 4.8 & $67 \%$ & 1.3 & 1.5 \\
\hline Verbal fluency & 34 & 40.8 & $50 \%$ & 38.3 & 37.2 & $50 \%$ & $56.6^{2}$ & 59.5 \\
\hline \multicolumn{9}{|l|}{ Memory and working memory } \\
\hline Visual span & $4.6^{2}$ & 5.8 & $60 \%$ & 5.3 & 5 & $50 \%$ & 8.3 & 7.8 \\
\hline Sentence span & $2.2^{2}$ & 2.3 & $60 \%$ & 1.8 & 2 & $17 \%$ & 3.7 & 3.3 \\
\hline Digit span & $7.4^{2}$ & 9.5 & $60 \%$ & 8.2 & 8.2 & $33 \%$ & 10.3 & 11.3 \\
\hline Dual span & 56.9 & 68.9 & $100 \%$ & 78.3 & 68.2 & $50 \%$ & 74.9 & 75.6 \\
\hline Generalised memory improvement ${ }^{1}$ & & 3 & & & 0 & & & \\
\hline
\end{tabular}

I. See Wykes et al (1999) for details of calculation.

2. Data available for only five participants.

Table 2 Regions identified in the analyses of covariance that are significantly different between patients and controls

\begin{tabular}{lcccc}
\hline Brain region & \multicolumn{2}{c}{ Talairach \& Tournoux (1988) coordinates } & $\begin{array}{c}\text { Brodmann } \\
\text { area }\end{array}$ \\
\cline { 2 - 4 } & $x$ & $y$ & $z$ & 47 \\
\hline Right inferior frontal gyrus & 43 & 25 & -7 & 46 \\
Left inferior/middle frontal gyrus & -38 & 44 & 4 & 11 \\
Right frontal cortex & 6 & 42 & -13 & 11 \\
Right frontal cortex & 9 & 47 & -13 & 10 \\
Left frontal (orbitofrontal) & -9 & 50 & -7 & 47 \\
Right insula/inferior frontal & 38 & 17 & 4 & $44 / 45$ \\
Right inferior frontal & 38 & 14 & -2 & 18 \\
Right occipital cortex (lingual gyrus) & 12 & -64 & -2 & 18 \\
Left occipital cortex (lingual gyrus) & -12 & -67 & 4 & - \\
Right insula & 38 & 14 & & \\
\hline
\end{tabular}

between the two therapy groups (see Table 2 for details of the individual results).

On the activation paradigms adopted in the scanner there was no difference between the patient and control groups on the identification of a target (mean per cent correct: patients 97\%, controls $95 \%$ ). However, the patients were less able to identify the target sequences in the working memory paradigm, although they were still performing above chance (mean per cent correct: patients $70 \%$, controls $99 \%$; $t=3.3, P<0.01)$ and there were no differences between the two patient groups.

\section{Changes over treatment phase}

After treatment there were no significant changes in the symptom and disability scores in either therapy group. However, there was a significant improvement in self-esteem $(t=2.61, P<0.05)$ in the CRT group.

There were no changes in the neuropsychological test scores of the healthy control participants (shown in Table 1) and there were no significant changes in the patient groups' detection rates for targets while in the scanner $(t=-0.22, P=0.9)$. Table 1 also gives data on the percentage of individuals in the two patient groups who improved by more than one standard error of the baseline scores (Wykes et al, 1999). Few differences were apparent on the cognitive flexibility tests. However, there was a definite advantage to the CRT group in the memory domain, where for three of the four tests more than twice as many people improved in the CRT group compared with the control therapy group. Half of the CRT group (i.e. $n=3$ ) showed an improved score from baseline to posttreatment on the tests of memory (i.e. showed an improved score on at least half of the tests).

\section{Functional magnetic resonance imaging data}

Following the statistical analysis set out above, the regions identified as differing between the groups at baseline using the analyses of covariance are shown in Table 2 . The location of the foci of these cerebral differences are referred to by their $x, y$ and $z$ coordinates, as set out by the Talairach \& Tournoux (1988) atlas, and by their Brodmann area positioning. All the differences were those in which there were higher numbers of activated voxels in the healthy control participant group than in either of the two patient participant groups. There were no differences between the two patient groups of any significance. These results indicate, as in most other studies of working memory, that patients with schizophrenia show less activation, especially in frontal regions.

\section{Changes over time: between and within groups}

A doubly multivariate repeated measures analysis was carried out with 'group' as the between-subject factor and 'time' as the within-subject factor, where all ten brain regions identified in Table 2 were tested simultaneously (i.e. to test the consistency of the changes across all brain regions using the Greenhouse-Geisser adjustment). 
Table 3 Results of the analyses of variance with contrasts

\begin{tabular}{|c|c|c|c|}
\hline Brodmann area & Dependent variable (brain region) & Group $\times$ time interaction & Significant post hoc tests (Tukey) \\
\hline 47 & Right inferior frontal gyrus & $F=4.70, P<0.026$ & Significant difference between CRT patients and healthy controls \\
\hline 46 & Left inferior/middle frontal gyrus & $F=4.41, P<0.031$ & $\begin{array}{l}\text { Significant difference between healthy controls and control therapy } \\
\text { patients }\end{array}$ \\
\hline II & Right frontal cortex & $F=5.39, P<0.017$ & $\begin{array}{l}\text { Significant difference between healthy controls and control therapy } \\
\text { patients }\end{array}$ \\
\hline 10 & Left frontal (orbitofrontal) & $F=3.87, P<0.044$ & Both patient groups significantly different from healthy controls \\
\hline 47 & Right insula/inferior frontal & $F=7.70, P<0.005$ & Both patient groups significantly different from healthy controls \\
\hline 18 & Right occipital cortex (lingual gyrus) & $F=5.40, P<0.017$ & Significant difference between CRT patients and healthy controls \\
\hline 18 & Left occipital cortex (lingual gyrus) & $F=5.34, P<0.018$ & Significant difference between CRT patients and healthy controls \\
\hline- & Right insula & $F=8.6, P<0.003$ & Both patient groups significantly different from healthy controls \\
\hline
\end{tabular}

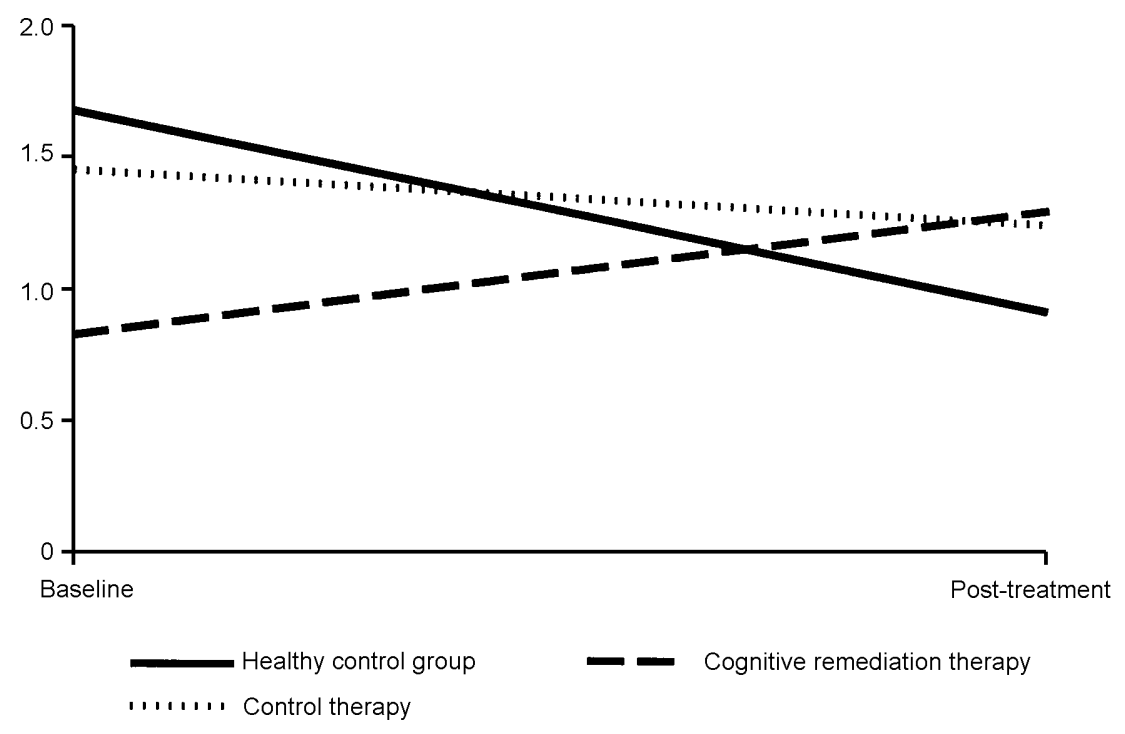

Fig. I Changes in fundamental power quotient (FPQ) values in the right inferior frontal gyrus.
The results of this analysis showed that there was no overall effect of group (Pillai's trace $F=2.59$, d.f. $11,6, P=0.127$ ) or time $(F=1.525$, d.f. $11,6, P=0.34)$ but the overall group $\times$ time interaction was significant $(F=2.41$, d.f. $11,6, P=0.046)$. Nine out of ten univariate tests had statistically significant interactions. Tukey post hoc tests were carried out on the change scores in the individual univariate tests and these significant contrasts are shown in Table 3.

The post hoc differences were investigated further using paired $t$-statistics. These showed that there were consistent changes for the CRT group in the right inferior frontal gyrus and left and right occipital cortex (lingual gyrus) (all showing increases in FPQ values from baseline to post-therapy). For the control therapy group there was a significant increase in the left frontal (orbitofrontal) and right frontal cortex. For the normal controls there were significant changes in the left inferior/middle frontal gyrus, right frontal cortex, right inferior frontal and right insula/inferior frontal, which were all in the direction of decreased activation post-therapy (see Table 2 for details of coordinates and Brodmann areas).

\section{Left visual cortex}

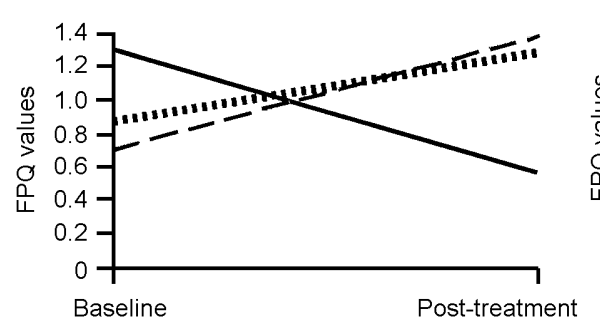

In order to illustrate these differences, Fig. 1 shows the activation change in the right inferior frontal gyrus, where the CRT group increased their activations, the control therapy group remained almost the same and the healthy controls decreased activation. Similar findings were found for the two occipital cortex measures, although here there were also some changes in the control therapy group (see Fig. 2).

The overall results of the FPQ analyses above show that there is a consistent increase in the power of the activation in the CRT group, whereas the normal control group reduced activation. In addition to the increase in activation in the frontal areas, there were also increases in the visual cortex for the patient groups. There were no differences in activation change between those who changed their medication and those who were on stable doses, nor between those on typical and those on atypical medication. On the whole, the changes in activation were for the healthy control group to decrease activation and for the patient groups to increase activation, with the CRT group increasing activation more consistently in key regions.

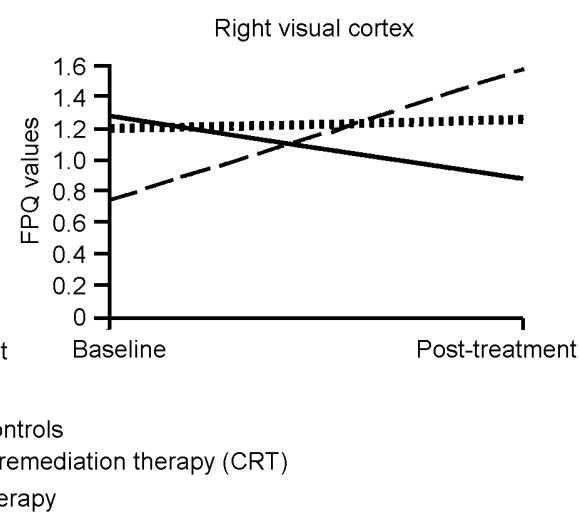

Fig. 2 Changes in fundamental power quotient (FPQ) values over the trial. 
Table 4 Location of the main focal regional cerebral changes over time for the three participants in the CRT group who improved on the neuropsychological memory measures

\begin{tabular}{lccccc}
\hline Cerebral region & $\begin{array}{c}\text { Number } \\
\text { of voxels }\end{array}$ & \multicolumn{2}{c}{ Talairach \& Tournoux (1988) coordinates } & $\begin{array}{c}\text { Brodmann } \\
\text { area }\end{array}$ \\
\cline { 3 - 6 } & & $x$ & $y$ & $z$ & \\
\hline Right prefrontal cortex & 12 & 40 & 14 & -2 & 47 \\
Left anterior temporal pole & 6 & -52 & 8 & -7 & 38 \\
Hippocampus & 6 & 9 & -44 & 4 & $30 / 19$ \\
Right post-cingulate gyrus & 6 & 17 & -69 & 9 & 31 \\
Right primary visual cortex & 4 & 3 & -58 & 4 & 18 \\
\hline
\end{tabular}

\section{Exploratory analyses of cognitive changes following CRT}

From the neuropsychological data collected outside the scanner it was clear that only three of the six participants had received any substantial benefit from the CRT (i.e. improved across the domain of memory tests). There were no initial differences between these three patients and the remaining three who received therapy. In particular, there were no differences in medication (one person in each of the two subgroups received the newer antipsychotic
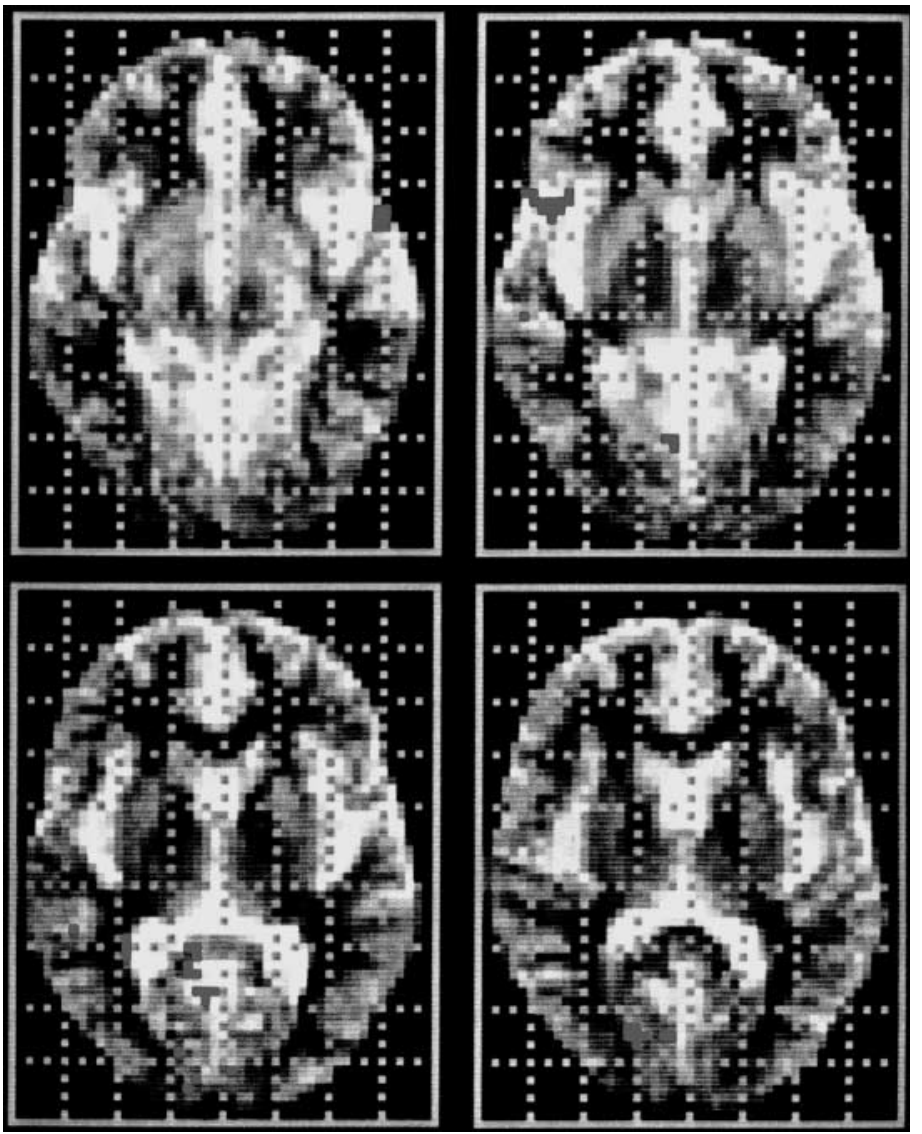

Fig. 3 Brain activation changes in patients with generalised cognitive improvements following cognitive remediation therapy. Red indicates areas of increased activation post-therapy (group images from the patients showing generalised cognitive improvements, at four slices).

change in activation in frontal and visua areas for these three successful patients. Red-coloured voxels indicated greater activation in the three CRT participants at post-treatment. The error threshold was set at 0.01 with a total of 51 voxels found to be significantly activated (two error voxels were expected). The details of the major changes over time are described in Table 4 .

The activation maps shown in Fig. 3 show that there is an increase in the size and number of activations. The number of generically activated voxels had risen from 43 to 133 (shown in red). The most prominent were identified around the frontal and visual cortical areas.

When further doubly multivariate repeated measures analyses were carried out on the FPQ values there were no significant effects of time, group or group $\times$ time interaction in the areas identified in Table 2 and no significant differential changes in the univariate tests. The only trend noted in the data was for the CRT improved group to decrease activation in Brodmann area 11 (right frontal cortex; $F=4.16, P<0.08$ ), which is similar to that noted in the normal control group.

\section{DISCUSSION}

The patients who participated in this study were very disabled. They had been in touch with psychiatric services for many years and were specifically chosen for the randomised trial because they had social functioning and cognitive difficulties. At baseline, the two groups of patients were very similar, although the CRT group had worse social functioning. The patient groups, as expected, performed significantly worse on all the neuropsychological tests. Over the course of the trial three patients, all in the CRT group, improved their performance on the memory tests that were performed outside the scanner. Neither symptoms nor disability changed significantly over the trial but self-esteem did improve for those patients who received CRT.

At baseline the results from the working memory activation scans replicated the results of many other studies, that is, there was significantly less activation in both patient groups compared with the healthy control group, particularly in the frontal and visual cortex (cf. Carter et al, 1998; Curtis et al, 1999; Honey et al, 1999; Wexler et al, 2000). This marked hypofrontality in patients with schizophrenia has been noted particularly in tasks with a marked working memory component (Callicott et al, 1999), despite the patients having similar characteristics to the healthy controls and despite making sure that the patients were carrying out the task to a similar level.

At post-treatment there were improvements in performance on the neuropsychological tasks, but improvements in memory were specifically related to receiving CRT, as was found in the larger study reported 
elsewhere (Wykes et al, 1999, 2002). At post-treatment there were also increases in activation in the areas that were significantly less activated in the healthy control group at baseline. Three patients in particular made generalised improvements (increased performance on all memory tests) and they had the largest activations.

The healthy control group changed little in performance on the memory tasks both in and outside the scanner. However, their brain activation generally decreased on the memory task at the time of the post-treatment scans. This change in activation with practice on the task has been noted by others (e.g. Goldman-Rakic, 1994). It is therefore even more surprising that the change in the patient groups was to increase their activation in key brain regions; this is particularly so in the CRT group. There were no other differences in clinical variables (e.g. medication or symptoms) that could account for the changes, so the most parsimonious explanation is that the CRT was responsible. Further evidence for this hypothesis comes from the association of marked increases in brain activity in those people who received the most benefit from the CRT.

\section{What do these data indicate about the hypofrontality in schizophrenia noted in previous studies?}

Previous studies that identified hypofrontality in patients with schizophrenia have generally been cross-sectional and, because of the multiple replications observed, hypofrontality has been assumed to be not only pervasive but also a trait phenomenon. However, this study has shown that it is neither a pervasive functional deficit nor a trait phenomenon. This has been pointed out by Curtis et al (1999), who found that hypofrontality was related to the type of task being carried out, with verbal fluency tasks showing more of this phenomenon than semantic decision tasks. But in that study it was not possible to identify the cause of these differences as there was little control over the behavioural response to the verbal fluency task, because it was covert, and the semantic decision task was easier. Either factor might have affected their results, in addition to any putative cognitive processing differences. In this longitudinal study the patients were presented with the same task so that the difficulty level was the same, the behavioural response was overt so that performance could be monitored and we must assume that the cognitive processing requirements were the same on each occasion. Therefore, the changes in activation patterns must be related to state rather than trait differences in cognition and brain activation.

\section{What is the likely mechanism for the changes in activation?}

There are two main issues here: to explain why healthy participants decrease activation on the second presentation of the working memory paradigm, which was 3 months later; and to explain why there were differential changes in the patient groups, particularly the CRT group. Activity in the frontal cortex could be related to a search for relevant information strategies that make the task simpler (or less effortful) to complete. One metaphor for this process would be the compiler setting up the schema for the contention scheduler in the model proposed by Shallice \& Burgess (1996), which may or may not be available to conscious examination. If this is the case, then practice will make access to a particular set of efficient schema more likely and the search can be terminated, producing smaller activations in particular brain regions. This might explain the decrease in the controls.

The increase for the patients could then be due to activating their search for a suitable contention schedule and associated schema on the second occasion. Both patient groups have been provided with one-to-one therapy and it might be expected that the effects of either therapy would be to increase attention during the task, but there were differential changes between the groups, particularly in prefrontal regions, that might be related to the specific therapy received. In neither type of therapy was the specific task practised. Cognitive remediation therapy concentrated on encouraging participants to adopt an organised approach to all tasks and particularly to increasing the use of specific information processing strategies. There is also an emphasis on rehearsal and other mnemonic strategies, which are useful in tasks requiring the storage and retrieval of material over both short and long (up to $10 \mathrm{~min}$ ) periods. It is possible that the changes in visual cortex are part of an improvement in using visual processing strategies in addition to verbal rehearsal. This might explain the lack of an advantage to the CRT group in the improvement on the visual span task.

Alternatively (or additively), both groups of patients receiving psychological therapy may have increases in their general attention, although other brain regions may then have been implicated that were not identified in the analyses presented here. It is also possible that increases in self-esteem lead to changes in self-efficacy, which lead to improvements in the pathways that are known to be used in this task. What is clear is that the improvements in neuropsychological task performance were produced by practice on information processing strategy choice and use, which is under conscious control.

The specific changes in activation patterns that were identified in this study were towards increases in regions that were identified as contributing to performance in the healthy control group and in other studies of working memory (Cohen et al, 1997). This is significant because there have been speculations that patients with schizophrenia may activate different brain regions in order to compensate for functional deficits. This was not apparent in the present study, although of course there may have been subtle activation that would not be found in such a small study. Smith \& Jonides (1998) suggest from their review that the left parietal cortex subserves the storage component of working memory and that left hemisphere speech areas subserve rehearsal. If this is the case (and there are many other regions implicated by other research groups), then neither of these areas are stimulated more than others in this study. This implies that the storage component is static or decreased in patients with schizophrenia, so that they must use more maintenance functions to increase their performance. This maintenance function has been suggested as being subserved by the prefrontal cortex (Cohen et al, 1997), which was one of the areas of increased activation at post-treatment. However, C. Frith (personal communication, 2002) considers activation in right prefrontal regions to be the result of monitoring for the appropriateness of the response. If this is the case, then participants with schizophrenia were not carrying out this self-monitoring before treatment but began monitoring afterwards.

Honey et al (1999) found similar results with another type of therapy, risperidone, which was associated with improvements in frontal cortex functioning over a shorter period of time. Although there are 
methodological difficulties with their study, particularly the lack of randomisation or blind assessment, the effects were to increase the frontal activation in those patients who were treated with atypical antipsychotic medication compared with controls. The effects of CRT cannot be explained as an effect of medication because three-quarters of the participants were prescribed typical antipsychotic medication and the type of medication was not correlated with the level of activation change. The differences achieved posttherapy in the Honey et al study were therefore achieved in the group that was least likely to improve. It may be that providing both psychological and atypical pharmacological therapy may produce an additive effect, but it is clear that psychological therapy with typical medication can produce such an effect.

\section{Was there a normalisation of activation following intervention?}

The activation patterns were significantly different from those of the healthy control participants before therapy but did not differ significantly after therapy. To this extent the patterns could be said to have normalised. However, they did not achieve the same levels of activation as the healthy participants at entry to the trial, but perhaps this is too stringent a criterion because the healthy participants decreased activation probably because of practice effects as well as reduced novelty. Both of these factors would also have affected the patient groups. Wexler et al (2000) suggest that it is possible to produce normalisation of performance following extended practice at a memory task, although their data are for only one patient.

In conclusion, this study has shown that cognitive performance changes following CRT are correlated with changes in brain activation, particularly increases in frontal regions. Recent studies have concentrated on the changes related to medication (Honey et al, 1999). However, there was no evidence that the changes measured either outside or inside the scanner were related to medication, although clearly there may be additive (or multiplicative) effects that can be identified only in a larger study. Our study demonstrates that hypofrontality may be ameliorated by psychological treatment. It remains to be seen whether such improvements in brain functions are sustained without further intervention or

\section{CLINICAL IMPLICATIONS}

Biological changes (brain activation) follow psychological therapy in chronic schizophrenia.

- A therapy with a high component of cognitive engagement - cognitive remediation therapy - increases activation in underactive behavioural and brain systems.

fMRI measures may be used as additional outcomes for psychological therapies in psychosis.

\section{LIMITATIONS}

- The sample size is small, so replication is required.

- Small but clinically significant effects may have been missed in the analyses.

- The patient participants were very disabled and might not be representative of all patients with schizophrenia.

TIL WYKES, PhD, MICHAEL BRAMMER, PhD, Institute of Psychiatry, London; JOHN MELLERS, MRCPsych, Maudsley Hospital, South London and Maudsley Trust; PETER BRAY, BSc, CLARE REEDER, DClinPsych, CLARE WILLIAMS, BA, JULIA CORNER, BA, Institute of Psychiatry, London

Correspondence: Professor Til Wykes, Department of Psychology, Institute of Psychiatry, De Crespigny Park, London SE5 8AF, UK. Tel: 0I7I 919 3596; e-mail: t.wykes@iop.kcl.ac.uk

(First received 13 March 200I, final revision 28 March 2002, accepted 16 April 2002)

whether 'top-up' or maintenance therapy is required. The research community has now amassed enough evidence that psychological therapy changes cognition in schizophrenia, and with the new evidence of accompanying brain changes we now have further hope of alleviating some of the misery associated with this disorder.

\section{ACKNOWLEDGEMENTS}

The authors thank the Wellcome Trust for supporting this MRI research and the Medical Research Council and Stanley Foundation for supporting the cognitive remediation trial.

\section{REFERENCES}

American Psychiatric Association (1994) Diagnostic and Statistical Manual of Mental Disorders (4th edn) (DSM-IV). Washington, DC: APA

Baddeley, A. (1986) Working Memory. Oxford: Oxford University Press.

_ \& Della Sala, S. (1996) Working memory and executive control. Philosophical Transactions of the Royal Society, London, Series B: Biological Sciences, 35I 1397-1404.
Brammer, M. J., Bullmore, E.T., Simmons, A., et al (1997) Generic brain activation mapping in $\mathrm{fMRI}$ : a nonparametric approach. Magnetic Resonance Imaging, I5, 763-770.

Bullmore, E.T., Brammer, M., Rabe-Hesketh, S., et al (1996) Statistical methods of estimation and inference for functional MR image analysis. Magnetic Resonance in Medicine, 35, 261-277.

_, _, _, et al (1999) Methods for diagnosis and treatment of stimulus-correlated motion in generic brain activation studies using fMRI. Human Brain Mapping, 7 , 38-48.

Burgess, P. \& Shallice, T. (1996) Response suppression initiation and strategy use following frontal lobe lesions. Neuropsychologia, 34, 263-273.

Callicott, J. H., Mattay, V. S., Bertolino, A., et al (1999) Physiological characteristics of capacity constraints in working memory as revealed by functional MRI. Cerebral Cortex, 9, 20-26.

Carter, C. S., Perlstein, W., Ganguli, R., et al (1998) Functional hypofrontality and working memory dysfunction in schizophrenia. American Journal of Psychiatry, I55, 1285-1287.

Cohen, J. D., Forman, S. D., Braver, T. S., et al (1994) Activation of the prefrontal cortex in a non-spatial working memory task with functional MRI. Human Brain Mapping, I, 293-304.

_, Perlstein, W. M., Braver, T. S., et al (1997)

Temporal dynamics of brain activation during a working memory task. Nature, 386, 604-608. 
Curtis, V. A., Bullmore, E.T., Morris, R. G., et al (1999) Attenuated frontal activation in schizophrenia may be task dependent. Schizophrenia Research, 37, 35-44.

Daneman, M. \& Carpenter, P. (1980) Individual differences in working memory and reading. Journal of Verbal Learning and Verbal Behaviour, 19, 450-466.

Delahunty, A. \& Morice, R. (1993) Training Programme for the Remediation of Cognitive Deficits in Schizophrenia. Albury, NSW: Department of Health.

Della Sala, S., Baddeley, A., Papagno, C., et al (1995) Dual task paradigm: a means to examine the central executive. Annals of the New York Academy of Sciences, 769, $|6|-17 \mid$.

Foster, P. (1989) Neuroleptic equivalence. Pharmacology Journal, 290, 431-432.

Goldman-Rakic, P. S. (1994) Working memory dysfunction in schizophrenia. Journal of Neuropsychiatry and Clinical Neuroscience, 6, 348-357.

Hogarty, G. E. \& Flesher, S. (1999) Developmental theory for a cognitive enhancement therapy of schizophrenia. Schizophrenia Bulletin, 25, 677-692.

Honey, G., Bullmore, E., Soni, W., et al (1999) Differences in frontal cortical activation by a working memory task after substitution of risperidone for typical antipsychotic drugs in patients with schizophrenia. Proceedings of the National Academy of Sciences of the USA, 96, 13432-13437.

Kindermann, S., Karini, A., Symonds, L., et al (1997) Review of functional magnetic resonance imaging in schizophrenia. Schizophrenia Research, 27, 143-156.
Mellers, J. D. C., Bullmore, E., Brammer, M., et al (1995) Neural correlates of working memory in a visual letter monitoring task: an fMRI study. Neuroreport, $\mathbf{7}$, 109-112.

Nelson, H. E. \& Willison, J. R. (199I) National Adult Reading Test (NART): Test Manual (2nd edn). Windsor: NFER-Nelson.

Rubin, P., Hemmingsen, R., Holm, S., et al (1994a) Relationship between brain structure and function in disorders of the schizophrenic spectrum: single positron emission computerized tomography, computerized tomography and psychopathology of first episodes. Acto Psychiatrica Scandinavica, 90, 28I-289.

_ , Holm, S., Madsen, P. L., et al (1994b) Regional cerebral blood flow distribution in newly diagnosed schizophrenia and schizophreniform disorder. Psychiatry Research, 53, 57-75.

Shallice, T. \& Burgess, P. (1996) The domain of supervisory processes and temporal organisation of behaviour. Philosophical Transactions of the Royal Society, London, Series B: Biological Sciences, 25I, 1405-1412.

Smith, E. F. \& Jonides, J. (1998) Neuroimaging analyses of human working memory. Proceedings of the National Academy of Sciences of the USA, 95, 12061-12068.

Spreen, O. \& Benton, A. (1977) Neurosensory Centre for the Comprehensive Examination of Aphasia (NCCEA) (revised edn). Melbourne: University of Victoria, Neuropsychological Laboratory.

Talairach, J. \& Tournoux, P. (1988) Co-Planar Stereotactic Atlas of the Human Brain. New York: Thieme Medical.
Trenerry, M., Crosson, B., De Boe, J., et al (1989) The Stroop Neuropsychological Screening Test. Odessa, FL: Psychological Assessment Resources.

Wechsler, D. (1981) Wechsler Adult Intelligence Scale Revised. New York: Psychological Corporation.

Weinberger, D. R., Berman, K. F. \& Zec, R. F. (1986) Physiologic dysfunction of dorsolateral prefrontal cortex in schizophrenia. I. Regional cerebral blood flow evidence. Archives of General Psychiatry, 43, 114-124.

Wexler, B., Anderson, M., Fulbright, R., et al (2000) Preliminary evidence of improved verbal working memory performance and normalization of task related to frontal lobe activation in schizophrenia following cognitive exercises. American Journal of Psychiatry, I57, 1694-1697.

Wykes, T. (1998) What are we changing with neurocognitive rehabilitation? Illustrations from two single cases of changes in neuropsychological performance and brain systems as measured by SPECT. Schizophrenia Research, 34, 77-86.

_ , Reeder, C., Corner, J., et al (1999) The effects of neurocognitive remediation on executive processing in patients with schizophrenia. Schizophrenia Bulletin, $\mathbf{2 5}$, 291-308.

_, _ Williams, C., et al (2002) Are the effects of cognitive remediation therapy (CRT) durable? Results from an exploratory trial. Schizophrenia Research, in press. 